\title{
Kritischer Blick auf die Prävention - wann ist weniger mehr?
}

Neuner-Jehle, S

\begin{abstract}
Vorbeugung kann Leiden und irreparable Schäden an der Gesundheit vermeiden helfen - kaum jemand wird die Logik und die Berechtigung präventiven Denkens und Handelns anzweifeln. Aber es gibt auch Schattenseiten: Prävention kann sich ungünstig auf die Balance zwischen Kosten und Nutzen und auf das Lebensgefühl auswirken. Im ersten Teil dieses Artikels versuchen wir, diese «Schatten auszuleuchten», im zweiten Teil werden konkrete Beispiele aus der kardiovaskulären Prävention präsentiert.
\end{abstract}

Posted at the Zurich Open Repository and Archive, University of Zurich ZORA URL: https://doi.org/10.5167/uzh-45522

Journal Article

Originally published at:

Neuner-Jehle, S (2010). Kritischer Blick auf die Prävention - wann ist weniger mehr? PrimaryCare, 10(19):363-368. 


\section{Kritischer Blick auf die Prävention - wann ist weniger mehr?}

\begin{abstract}
Vorbeugung kann Leiden und irreparable Schäden an der Gesundheit vermeiden helfen - kaum jemand wird die Logik und die Berechtigung präventiven Denkens und Handelns anzweifeln. Aber es gibt auch Schattenseiten: Prävention kann sich ungünstig auf die Balance zwischen Kosten und Nutzen und auf das Lebensgefühl auswirken. Im ersten Teil dieses Artikels versuchen wir, diese "Schatten auszuleuchten", im zweiten Teil werden konkrete Beispiele aus der kardiovaskulären Prävention präsentiert.
\end{abstract}

Vorbeugung kann Leiden und irreparable Schäden an der Gesundheit vermeiden helfen - kaum jemand wird die Logik und die Berechtigung präventiven Denkens und Handelns anzweifeln. Kein Zufall also, dass sich die Grundversorgenden damit identifizieren, sich nebst ihren vielen beruflichen Aufgaben auch in der Prävention zu engagieren. Diese ist gemäss Wonca-Definition eine Kernkompetenz der Hausärzte: «Die Allgemeinmedizin fördert Gesundheit und Wohlbefinden durch angemessene und wirksame Intervention» und verfügt über «die Fähigkeit, Gesundheit und Wohlbefinden durch Gesundheitsförderungs- und Krankheitspräventionsstrategien in geeigneter Weise zu fördern» sowie "Gesundheitsförderung und Prävention (...) zu betreiben und zu koordinieren» [1]. Laut Text unserer aktuellen Volksinitiative «Ja zur Hausarztmedizin» sind Grundversorgende erste Anlaufstelle für die Behandlung von Krankheiten und Unfällen, aber auch «für Fragen der Gesundheitserziehung und Gesundheitsvorsorge» und dafür angemessen zu entlöhnen [2].
Erfolgreich umgesetzte Präventionsstrategien können die Krankheitslast einer Bevölkerung reduzieren - dies hat zur Etablierung von Public Health als Fachdisziplin geführt, und auch die Industrie hat längst entdeckt, dass Prävention lukrativ sein kann. Wo aber sind die Schattenseiten?

\section{Prävention konkurriert kurative Medizin}

Für die Prävention gelten die gleichen Regeln wie für andere medizinische Interventionen: Der zu erwartende Nutzen muss das Risiko ungünstiger Wirkungen überwiegen. Und weil Prävention ebenso wie kurative Medizin kostet, muss auch sie auf ihre Kosteneffizienz geprüft werden [3].

Number needed to treat und seine Konfidenzintervalle

Eine nützliche Grösse, Interventionen bezüglich Nutzen, Schaden und Kosten miteinander zu vergleichen, ist die «number needed to treat» (NNT), die Anzahl Patienten also, die über einen definierten Zeitraum behandelt werden muss, damit ein Patient von der Behandlung profitiert (d.h. einen definierten Endpunkt des Behandlungserfolges erreicht). In der Primärprävention sind die NNT oft im dreistelligen Bereich, während sie in der kurativen Medizin (glücklicherweise) oft im tiefen einstelligen Bereich liegen: Die Behandlung von Schmerzen etwa erlaubt nur eine sehr tiefe NNT, etwa 2 bis 3, um noch akzeptabel zu sein, ansonsten würde niemand ein solches (zu wenig wirksames) Analgetikum einsetzen. Sind präventive Interventionen kostengünstig und mit wenig Aufwand oder Schadenspotential verbunden, stört eine hohe NNT nicht so sehr, als wenn sie kostspielig, aufwendig oder nebenwirkungsreich sind.

Tabelle 1

NNT für einige sekundärpräventive Interventionen kardiologischer Natur.

ARR = absolute Risikoreduktion durch die Intervention; die Quellenangaben zu den einzelnen Studien finden sich bei [4].

\begin{tabular}{|c|c|c|c|c|c|c|}
\hline Diagnose & Intervention & Outcome & Dauer (Jahre) & ARR (\%) & NNT $(95 \%-\mathrm{Cl})$ & $95 \%-\mathrm{Cl}$ der NNT \\
\hline $\begin{array}{l}\text { Koronare Ereignisse bei } \\
\text { Gesunden mit tiefem HDL }\end{array}$ & $\begin{array}{l}\text { Lovastatin } \\
\text { vs. } \\
\text { Placebo }\end{array}$ & $\begin{array}{l}\text { Erstmalige } \\
\text { koronare } \\
\text { Ereignisse }\end{array}$ & 5,2 & 2,0 & 50 & 33-97 \\
\hline $\begin{array}{l}\text { Kardiovaskuläre Ereignisse } \\
\text { bei behandelter Hypertonie }\end{array}$ & $\begin{array}{l}\text { Aspirin } \\
\text { vs. } \\
\text { Placebo }\end{array}$ & $\begin{array}{l}\text { Kardiovask. } \\
\text { Ereignisse } \\
\text { Myokardinfarkt }\end{array}$ & $\begin{array}{l}3,8 \\
3,8\end{array}$ & $\begin{array}{l}0,6 \\
0,5\end{array}$ & $\begin{array}{l}176 \\
208\end{array}$ & $\begin{array}{l}90-3115 \\
127-551\end{array}$ \\
\hline $\begin{array}{l}\text { Ältere Menschen mit } \\
\text { durchschnittlichen } \\
\text { Cholesterinwerten }\end{array}$ & $\begin{array}{l}\text { Pravastatin } \\
\text { vs. } \\
\text { Placebo }\end{array}$ & $\begin{array}{l}\text { Koronare } \\
\text { Ereignisse } \\
\text { CVI } \\
\text { Tod an KHK } \\
\text { Bypass-OP }\end{array}$ & 5 & $\begin{array}{l}8,4 \\
\\
2,8 \\
4,5 \\
4,4\end{array}$ & $\begin{array}{l}11 \\
34 \\
22 \\
21\end{array}$ & $\begin{array}{l}8-24 \\
22-333 \\
15-53 \\
15-56\end{array}$ \\
\hline Chronische Herzinsuffizienz & $\begin{array}{l}\text { Physikalische } \\
\text { Aktivität vs. } \\
\text { keine Aktivität }\end{array}$ & $\begin{array}{l}\text { Tod } \\
\text { Kardiale Ereignisse } \\
\text { Hosp. wegen } \mathrm{HI}\end{array}$ & 3,4 & $\begin{array}{l}22,8 \\
41,5 \\
18,6\end{array}$ & $\begin{array}{l}5 \\
3 \\
6\end{array}$ & $\begin{array}{l}3-21 \\
2-5 \\
3-32\end{array}$ \\
\hline $\begin{array}{l}\text { Erwachsene mit hohem } \\
\text { kardiovaskulärem Risiko }\end{array}$ & $\begin{array}{l}\text { Ramipril } \\
\text { vs. Placebo }\end{array}$ & $\begin{array}{l}\text { Myokardinfarkt, } \\
\text { CVI und kardiovaskuläre } \\
\text { Mortalität }\end{array}$ & 4 & 4,0 & 26 & $19-43$ \\
\hline
\end{tabular}


Die Website der kanadischen Evidence-Based-Mediziner gibt eine schöne Übersicht über die NNT häufiger primärpräventiver, sekundärpräventiver und kurativer Interventionen [4]; in Tabelle 1 findet sich eine Auswahl davon:

Zu Recht weisen die Autoren darauf hin, dass auch NNT ihre Konfidenzintervalle (Cl) haben, genauso wie die Daten (Differenzen absoluter Prozentwerte und Odds Ratios), von denen sie hergeleitet sind. Wie sollen wir diese Aufstellungen interpretieren? Betrachten wir die erste Zeile der Tabelle 1: Um ein koronares Ereignis bei Gesunden mit einem tiefen HDL zu vermeiden, muss man deren 50 während über fünf Jahren mit Lovastatin behandeln (NNT $=50$ ). Diese Zahl erscheint mir so gerade akzeptabel. Das 95\%-Konfidenzintervall für diese NNT beträgt 33 bis 97: Ist es immer noch akzeptabel, 97 Gesunde mit tiefem HDL während fünf Jahren mit einem relativ teuren Medikament zu behandeln und sie potentiellen $\mathrm{Ne}$ benwirkungen auszusetzen, damit ein einziges koronares Ereignis vermieden wird? Oder sehen Sie sich die zweite Zeile der Tabelle 1 an: Wie beurteilen Sie die Effizienz einer Aspirin-Sekundärprophylaxe bei Hypertonikern hinsichtlich kardiovaskulärer Ereignisse? Natürlich schleichen sich Zweifel ein, ob die Balance zwischen Nutzen, Kosten und Schaden in solchen Fällen stimmt.

Prävention ja - aber durch wen?

Nicht nur beim Verbrauch von finanziellen Ressourcen, sondern auch in der konkurrierenden Beanspruchung von Medizinalpersonen ist Prävention nicht unproblematisch: Die Zeit, die wir Grundversorger dafür aufwenden, fehlt uns in der (kurativen, palliativen) Betreuung unserer erkrankten Patienten. In einem sehr lesenswerten Artikel [3] werfen Michael Weingarten und André Matalon die Frage auf, ob es ethisch vertretbar ist, die Hausärztin bzw. den Hausarzt in zunehmendem Ausmass mit präventiven Aufgaben zu betrauen und damit zu absorbieren. Yarnall et al. stellten fest, dass das Befolgen aller Guidelines für Prävention 7,4 Stunden pro Arbeitstag beanspruchen würde - selbst wenn wir dies auf europäische Verhältnisse adaptieren, wäre immer noch der halbe Arbeitstag mit Aufgaben der Prävention belegt [5]. Sind die wesentlichen präventiven Aktivitäten einer Hausarztpraxis wie Impfen, (kardiovaskuläre) Risiken erfassen, gesunden Lebensstil fördern, Screening und Früherfassung von asymptomatischen Krebserkrankungen wirklich nur durch uns Ärzinnen und Ärzte zu leisten, die jahrzehntelang darauf trainiert wurden, exakte Diagnosen zu stellen und adäquate Therapien durchzuführen? Oder sollten wir diese Aufgaben - unter ärztlicher Anleitung - für unsere Praxisassistentinnen öffnen, nach einer spezifischen (aber nicht jahrzehntelangen) Schulung in gesundheitsfördernden und präventiven Themen?

\section{Prävention mit Fokus auf Risiken erzeugt Angst, stigmatisiert und medikalisiert}

Im Gegensatz zum Wirksamkeitsnachweis von Public-Health-Interventionen, der mit viel Aufwand geführt und publiziert wird, besteht ein beunruhigendes «schwarzes Loch» in der Forschung, wenn es um die negativen Auswirkungen von Kampagnen und anderen präventiven Aktivitäten geht [6]. Die Erfahrung zeigt aber: Die Beschäftigung mit Risiko kann fast obsessive Ausmasse annehmen und eine «Angstkultur» erzeugen, in der die Angst instrumentalisiert wird, um neue Krankheiten zu generieren (disease mongering) und Gewinne mit deren Behandlung einzufahren. Wer von Ihnen, die in der Praxis arbeiten, hat noch keine nächtlichen Telefonate von besorgten Hypertonikern erhalten, die im Stundentakt erhöhte Blutdruckwerte gemessen hatten? Wer kennt sie nicht, die Patienten, die monatlich ihre Cholesterinwerte oder sechs Mal täglich Blutzucker messen? Ergibt sich ein direkter Nutzen durch die Check-up-Laboranalysen eines 30-Jährigen, der sich gesund fühlt und weder familiäre Risiken mitbringt noch einen ungesunden Lebensstil hat? ${ }^{1}$ Das Sicherheitsbedürfnis, die schwindende Bereitschaft, Risiken auszuhalten, hält eine ganze Medizinindustrie am Leben. «Get scanned!», lautete eine US-Schlagzeile aus den 1970er Jahren, gönnen Sie sich eine Ganzkörpercomputertomografie! Heute übersetzt sich der Slogan in «Get screened!» und versorgt Radiologen, Gastroenterologen, Gynäkologen, Urologen und Präventivmediziner mit Arbeit und Sold. So sind wir Mediziner und Medizinerinnen keineswegs unschuldig an dieser Entwicklung.

Diejenigen, die sich der Maxime der Risikofreiheit nicht beugen wollen oder können, geraten unter gesellschaftlichen Druck, falls sie an einer vorzubeugenden Krankheit erkranken, oder sie werden stigmatisiert [7], zum Beispiel beim Zugang zu Kranken- und Lebensversicherungen. Die Maxime, Risiken weitmöglichst zu vermeiden, hat etwas genussfeindliches an sich, antizipiert Krankheit und kann bei unklaren, wahrscheinlich unbedeutenden Untersuchungsbefunden die Unsicherheit bei Arzt und Untersuchtem verstärken und «sicherheitshalber» unnötige Nachuntersuchungen auslösen. Die Grillbratwurst mutiert zur Kanzerogenbombe, der Sommer zur Melanomsaison, ein gemütlicher Lesenachmittag zur verwerflichen körperlichen Inaktivität [8]. Das zur Sicherheit durchgeführte CT wird zum Startschuss für eine ganze Serie von «Sicherheits-KontrollCTs», die Diagnostikmaschinerie perpetuiert. Der südamerikanische Poet Jorge Luis Borges hat es so ausgedrückt [9]:

\section{Wenn ich mein Leben noch einmal leben könnte,} im nächsten Leben würde ich versuchen, mehr Fehler zu machen. Ich würde nicht so perfekt sein wollen, ich würde mich mehr entspannen.

Ich wäre ein bisschen verrückter, als ich es gewesen bin, ich würde viel weniger Dinge so ernst nehmen.

Ich würde nicht so gesund leben.

Ich würde mehr riskieren, würde mehr reisen, Sonnenuntergänge betrachten, mehr bergsteigen, mehr in Flüssen schwimmen.

Ich war einer dieser klugen Menschen, die jede Minute ihres Lebens fruchtbar verbrachten. Freilich hatte ich auch Momente der Freude.

Aber wenn ich noch einmal anfangen könnte, würde ich versuchen, nur mehr gute Augenblicke zu haben. Falls du es noch nicht weisst, aus diesen besteht nämlich das Leben. Nur aus Augenblicken. Vergiss nicht den jetzigen.

Wenn ich noch einmal leben könnte, würde ich von Frühlingsbeginn an bis in den Spätherbst barfuss gehen. Und ich würde mehr mit Kindern spielen, wenn ich das Leben noch vor mir hätte.

Aber sehen Sie; ich bin 85 Jahre alt und weiss, dass ich bald sterben werde.

Auch neue, emfohlene Methoden, wie Risiko verständlich dargestellt werden kann, sind vor solchen angsterzeugenden «Nebenwirkungen» nicht gefeit: Wenn beispielsweise ein 50-jähriger Raucher darüber aufgeklärt wird, dass seine (spirometrisch gemessene) Lungenfunktion infolge des Rauchens der Lungenfunktion eines gesunden 70-jährigen entspricht, unterstützt das vielleicht seine Motivation, das Rauchen aufzugeben, löst aber auch Ängste aus

Zur Ehrenrettung der Check-up-Untersuchung muss bemerkt werden, dass sie ihren Nutzen daraus schöpft, dass der Patient in diesem Rahmen Gelegenheit bekommt, seine «versteckte Agenda» anzusprechen und mit der Ärztin oder dem Arzt seines Vertrauens ins Gespräch darüber zu kommen. 
[10]. Wie würden Sie empfinden, wenn ich Ihnen eröffne, dass Ihr Herz - bezogen auf das Risiko, in den nächsten fünf Jahren zu infarzieren, und im Vergleich mit einem gesunden Menschen - 20 Jahre älter sei als Sie selbst an Lebensjahren? Wohl nicht gerade eine Erkenntnis, die Sie ruhig schlafen lässt.

\section{Die neue Disziplin: quartäre Prävention}

Aus der Zielsetzung heraus, die «besorgten Gesunden (worried well)» vor unnötigen medizinischen Interventionen und deren potentiellen Schäden zu schützen, hat sich eine eigene Disziplin entwickelt: die quartäre Prävention [11]. Sie will - in Analogie zur Primär- und Sekundärprävention (Vermeiden ungesunder Entwicklungen bei Gesunden und Risikoträgern) und der Tertiärprävention (dito bei manifest Erkrankten) - Gesunde, die sich krank oder eben besorgt fühlen, vor unnötigen oder schädlichen Präventionen, Abklärungen oder Behandlungen bewahren. Voraussetzungen dafür sind evidenzbasierte Daten und eine vertrauensvolle Arzt-Patienten-Beziehung. Solche Themen werden in hausärztlichen Fachkreisen zunehmend diskutiert, so auch am Wonca-Kongress 2009 in Basel [12].

Ein konkretes Beispiel, wo sich quartäre Prävention lohnt, ist das wiederholte Überprüfen der Medikation von älteren polymorbiden Menschen auf ihre Indikation und ihr Schadenpotential hin, also das Vermeiden der Polypharmazie mit ihrem Interaktionspotential: So ergab eine britische Studie, dass ein Pharmazeut während acht Monaten die Medikamentenliste von 17 Altersheimbewohnern regelmässig durchsehen und bereinigen musste, um einen Todesfall durch ein adverses, medikamenteninduziertes Ereignis zu verhindern [13]. Aufmerksame Leser fragen natürlich, wie denn bei dieser NNT von 17 das 95\%-Konfidenzintervall aussieht. Zuge geben, mit einem deklarierten Intervall zwischen 9 und 213 ist diese NNT nicht über alle Zweifel erhaben [13].

Wirken sich präventive Aktivitäten beim Hausarzt auf andere wichtige Aufgaben verdrängend aus, ist die Reduktion oder der Verzicht darauf eine vertiefte Reflexion wert. Wenn beim Patienten durch ausführliche Beschäftigung mit seinem Risiko Ängste oder Zwänge erzeugt werden, ist das ärztliche Gespräch sinnvoll, jedoch Zurückhaltung mit medizinanalytischen und technischen Untersuchungen angesagt. Nur weil das Konzept der Prävention an sich sinnvoll ist, muss die Balance zwischen Kosten und Nutzen oder zwischen Nutzen und Schaden bei präventiven Aktivitäten nicht automatisch stimmig sein. Manchmal kann auch bei der Prävention weniger mehr sein - anstelle eines «vorauseilenden Gehorsams» gegenüber Guidelines und Empfehlungen sind eine kritische Grundhaltung unsererseits und die Mitsprache des Patienten zielführend. Beherzigen wir auch hier: primum non nocere!
Teil 2: Die kardiovaskuläre Prävention und ihre Problemzonen Nachdem sich der erste Teil des Artikels mit den ungünstigen Folgen der Prävention auf die Kosten-Nutzen-Balance und das Lebensgefühl beschäftigt hat, wollen wir diese kritischen Überlegungen anhand von Beispielen aus der kardiovaskulären Prävention konkretisieren. Die Herz-Kreislauf-Prävention ist ein Klassiker der präventiven Medizin und eine Erfolgsgeschichte. Dennoch tun sich viele Hausärztinnen und -ärzte schwer, die Guidelines der Spezialisten dazu strikt zu befolgen. Einer der Gründe dafür ist die Ungleichheit der Populationen: Guidelines stützen sich in der Regel auf Daten aus Studienpopulationen, die an einer Krankheit leiden (oder Risiken dafür tragen) und dagegen behandelt werden. Unsere Praxispatienten sind aber oft multimorbide und erhalten dementsprechend multiple Interventionen/Medikamente, so dass die Leitlinien für eine einzelne Krankheit nicht ohne weiteres auf unsere Patienten übertragen werden können. Aber auch an der NutzenSchaden-Balance der Interventionen selbst sind manchmal Zweifel angebracht; die Leitlinien dann nicht einzuhalten, kann sich in solchen Fällen sogar als sinnvoll erweisen.

\section{Der abnehmende Grenznutzen - welche Intervention bringt den grössten Mehrwert?}

An einem kürzlichen Seminar am «Prevention Summit» des Unispitals Zürich über Risikokommunikation bemerkte ein Teilnehmer, dass die grösste Wirkung bei kardiovaskulärer Prävention wohl die Blutdrucksenkung entfalte - immer, wenn er mit dem Risikokalkulator berechne, um wie viel das Risiko des Patienten durch die Blutdruckregulation sinke, dann erzielten alle weiteren Massnahmen wie Lipidsenkung oder Rauchstopp vergleichsweise bescheidene Werte. Stimmt das? Machen wir die Probe aufs Exempel und nehmen einen 62-jährigen Raucher mit einem Blutdruck von 180/90 mm Hg und einem Cholesterin von 6,5 mmol/l, Triglyceriden von $2,2 \mathrm{mmol} / \mathrm{l}$, einem $\mathrm{HDL}$ von $1,0 \mathrm{mmol} / \mathrm{l}$ und einem $\mathrm{LDL}$ von $4,5 \mathrm{mmol} / \mathrm{I}$. Die Berechnung mittels AGLA-Score [26] ergibt ein Risiko von $40 \%$, in den nächsten zehn Jahren kardiovaskulär zu erkranken. Durch realistische Interventionen in seinen drei Risikobereichen lässt es sich auf 12,8\% senken (Tab. 2).

Abhängig davon, mit welcher Intervention man beginnt, könnten die Werte aber auch anders ausfallen (Tab. 3, 4).

Wie unschwer erkennbar ist, sind bei identischen Ausgangs- und Zielwerten (nach allen drei Interventionen) die einzelnen Interventionen je nach Reihenfolge ihres Einsatzes verschieden bewertet, was die Reduktion des absoluten Risikos betrifft. Tabelle 2 gibt dem aufmerksamen Seminarteilnehmer recht, indem die Blutdrucksenkung der Lipidsenkung überlegen scheint (bei gleichzeitig starkem Auftritt des Rauchstopps). In Tabelle 3 überflügelt die Cholesterin-

\section{Tabelle 2}

Zehn-Jahres-Morbiditätsrisiko für ein kardiovaskuläres Ereignis. Gemäss AGLA-Score [26] mit der Interventionsreihenfolge Blutdrucksenkung - Lipidsenkung - Rauchstopp.

\begin{tabular}{|c|c|c|c|c|}
\hline Intervention & Absolutes Risiko & Relative Risikoreduktion & Absolute Risikoreduktion & NNT \\
\hline Blutdruck 180/90 mm Hg, LDL 4,5 mmol/l, Raucher (Ausgangswert) & $40,0 \%$ & - & - & - \\
\hline Senkung des BD auf 140/85 mm Hg & $30,3 \%$ & $24 \%$ & $9,7 \%$ & 10 \\
\hline Senkung des LDL auf $3,75 \mathrm{mmol} / \mathrm{l}$ & $22,6 \%$ & $25 \%$ & $7,7 \%$ & 13 \\
\hline Rauchstopp & $12,8 \%$ & $43 \%$ & $9,8 \%$ & 10 \\
\hline
\end{tabular}


Tabelle 3

Zehn-Jahres-Morbiditätsrisiko für ein kardiovaskuläres Ereignis. Gemäss AGLA-Score [26] mit der Interventionsreihenfolge Lipidsenkung - Blutdrucksenkung - Rauchstopp.

\begin{tabular}{|c|c|c|c|c|}
\hline Risiko & Absolutes Risiko & Relative Risikoreduktion & Absolute Risikoreduktion & NNT \\
\hline Blutdruck 180/90 mm Hg, LDL 4,5 mmol/l, Raucher (Ausgangswert) & $40,0 \%$ & - & - & - \\
\hline Senkung des LDL auf 3,75 mmol/l & $30,8 \%$ & $23 \%$ & $9,2 \%$ & 11 \\
\hline Senkung des BD auf 140/85 mm Hg & $22,6 \%$ & $27 \%$ & $8,2 \%$ & 12 \\
\hline Rauchstopp & $12,8 \%$ & $43 \%$ & $9,8 \%$ & 10 \\
\hline
\end{tabular}

\section{Tabelle 4}

Zehn-Jahres-Morbiditätsrisiko für ein kardiovaskuläres Ereignis. Gemäss AGLA-Score [26] mit der Interventionsreihenfolge Rauchstopp - Blutdrucksenkung - Lipidsenkung.

\begin{tabular}{|c|c|c|c|c|}
\hline Risiko & Absolutes Risiko & Relative Risikoreduktion & Absolute Risikoreduktion & NNT \\
\hline Blutdruck 180/90 mm Hg, LDL 4,5 mmol/l, Raucher (Ausgangswert) & $40,0 \%$ & - & - & - \\
\hline Rauchstopp & $24,8 \%$ & $38 \%$ & $15,2 \%$ & 7 \\
\hline Senkung des BD auf 140/85 mm Hg & $17,8 \%$ & $28 \%$ & $7,0 \%$ & 14 \\
\hline Senkung des LDL auf 3,75 mmol/l & $12,8 \%$ & $28 \%$ & $5,0 \%$ & 20 \\
\hline
\end{tabular}

senkung als Erstintervention die Blutdrucksenkung, und in Tabelle 4, wo der Rauchstopp die erste und die Cholesterinsenkung die letzte Massnahme ist, glänzt der Rauchstopp mit einer doppelt bis dreifach erhöhten absoluten Risikoreduktion im Vergleich zu den beiden anderen Massnahmen. Anhand der relativen Prozente der Risikoreduktion lässt sich die Überlegenheit des Rauchstopps übrigens gut erkennen.

Die erste Intervention erhält also gegenüber allen weiteren einen scheinbaren «Bonus», der sich durch das höhere Ausgangsrisiko vor Intervention erklärt - dieses Ausgangsrisiko sinkt vor jeder zusätzlichen Intervention. Der Zusammenhang lässt sich mit dem Begriff Grenznutzen illustrieren: Mit steigendem Aufwand steigt der Nutzen nicht linear an; der zusätzliche (inkrementale) Nutzen wird im Verhältnis dazu immer kleiner (Abb. 1).

Ausnahmen können besonders wirksame Interventionen - wie in unserem Beispiel der Rauchstopp - darstellen, dann würde die Aufwand-Nutzen-Kurve nicht abgeflacht verlaufen, sondern einen Sprung nach oben machen.

Der Begriff Grenznutzen stammt eigentlich aus der Volkswirtschaftslehre, wo das Erste Gossen'sche Gesetz (Gesetz vom abnehmenden Grenznutzen) von 1854 besagt: «Die Grösse ein und desselben Genusses nimmt, wenn wir mit der Bereitung des

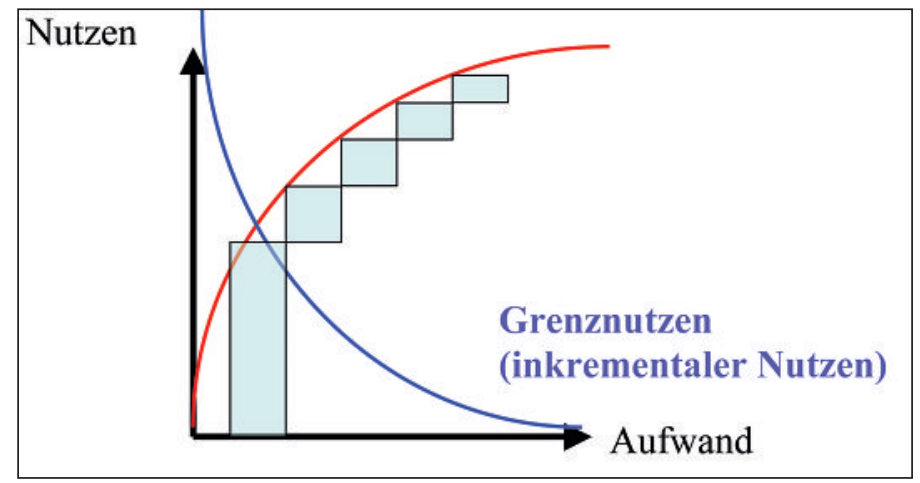

Abbildung 1

Verhältnis von Nutzen zu Aufwand, Begriff des Grenznutzens.
Genusses ununterbrochen fortfahren, fortwährend ab, bis zuletzt Sättigung eintritt» [14]. Machen Sie einen Selbstversuch: Der abnehmende Grenznutzen wird direkt erfahrbar, wenn Sie durstig sind und nacheinander immer das gleiche Volumen Wasser trinken - Genuss (und Nutzen) nimmt ab.

\section{Gibt es eine optimale Reihenfolge kardiovaskulärer Interventionen?}

Das Bewusstsein um den Grenznutzen und die Bedeutung des Ausgangsrisikos bzw. der Reihenfolge der Interventionen ist wichtig, um - angesichts knapper Ressourcen - beim Priorisieren von Interventionen nicht Fehlschlüsse über deren Wirksamkeit zu ziehen. Wenden wir uns nochmals unserem Modellpatienten zu: Entsprechend unserer Praxisrealität postulieren wir, dass er nicht bereit ist, sofort mehrere Interventionen auf sich zu nehmen. Er akzeptiert eine, höchstens zwei Änderungen seines Verhaltens oder Medikamente. Zu welcher Intervention sollen wir ihm raten, um ein Maximum an Wirkung zu erzielen (zur Vereinfachung angenommen, dass die drei möglichen Interventionen ungefähr gleich wirksam und gleich teuer seien)? Tabellen 2 bis 4 zeigen, dass die Blutdruckund Lipidsenkung als Erstintervention (bzw. als einzige Intervention) mit 9,7 bzw. 9,2\% dem Rauchstopp mit 15,2\% deutlich unterlegen sind. Somit lautet die Empfehlung: Beginnen Sie bei Rauchern immer mit dem Rauchstopp. Auch auf der Kostenseite lohnt der initiale Aufwand, da im Gegensatz zu einer langfristigen antihypertensiven oder lipidsenkenden Medikation keine Dauerkosten anfallen. Ist unser Patient zu einer weiteren Intervention bereit, ist die Blutdrucksenkung die zweiteffizienteste Massnahme (Tab. 4).

Berechnen Sie bei Ihrem nächsten Patienten, der multiple kardiovaskuläre Risiken trägt, analoge Zahlen, und zeigen Sie ihm den «Wert» der Erstintervention auf - Sie werden staunen, wie viel zusätzliche Motivation dies erzeugt.

Die Tabellen 2 bis 4 zeigen aber auch, dass bei der Hochrisikokonstellation unseres Modellpatienten die NNT mit Werten zwischen 7 und 20 für sekundärpräventive Verhältnisse sehr tief sind und sich alle drei Interventionen lohnen dürften (zumindest bezüglich Morbidität). Anders könnte es im folgenden Beispiel aussehen. 


\section{Kardiovaskuläre Prävention im Alter:} bescheidene Wirkung, erhebliche Nebenwirkungen?

Betreuen Sie auch ältere Patienten mit Merkmalen wie bei der folgenden Fallgeschichte? Frau M. ist 92 Jahre alt und hat vor drei Jahren einen cerebrovaskulären Insult mit partieller Resthemiplegie erlitten, der sie zum Eintritt ins Alters- und Pflegeheim gezwungen hat. Zuvor war sie mit Ausnahme einer mässigen, behandelten arteriellen Hypertonie recht rüstig gewesen. Während der Hospitalisation ist man auf der Suche nach kardiovaskulären Risikofaktoren fündig geworden: eine moderate Dyslipidämie (Gesamt-Cholesterin 6,5 mmol/l, HDL 1,2 mmol/l, LDL 4,2 mmol/l). Unter dem Eindruck des CVI bekommt sie nebst dem obligaten Aspirin cardio als Tertiärprophylaxe ein Statin verordnet. Sinnvoll oder überflüssig? Die Kosten-Nutzen-Abwägung zum Einsatz eines Statins bei dieser Patientin könnte so geführt werden: Bei jüngeren Patienten ist die Indikation gemäss den Richtlinien der Kardiologengesellschaften gegeben. Rechtfertigt ihr hohes Alter den Verzicht darauf? Angesichts ihres bisher rüstigen Gesundheitszustandes kann ihre Restlebenserwartung auf etwa fünf bis sechs Jahre geschätzt werden [15], und selbst unter Einbezug des CVI ergibt der Charlson-Index ${ }^{2}$ [16] eine Wahrscheinlichkeit von 75\%, dass sie den Grossteil des nächsten Lebensjahrzehntes noch erlebt. Das Statin könnte ihr ermöglichen, diese Jahre bei besserer Gesundheit zu verbringen, wenn es damit gelänge, ein cererbrales Rezidivereignis zu verhindern. Betrachten wir aber, was die PROSPER-Studie (mit einer untersuchten Population bis Lebensalter 82 Jahre) über die Wirkung von Statinen im Alter ergab [17], so zeigt sich, dass bei älteren Patienten mit kardiovaskulärer Erkrankung oder hohem kardiovaskulärem Risiko eine Statinbehandlung während dreier Jahre zu 2,1\% weniger kardiovaskulären Ereignissen führt (kombinierter Endpunkt). Lohnt sich der Einsatz wirklich bei dieser NNT von 50? Und wenn wir aus der genannten Studie noch wissen, dass für den Endpunkt CVI keine signifikante Differenz mehr gefunden wurde zwischen Interventions- und Kontrollgruppe, verstärkt das die Zweifel noch. Eine neue Metaanalyse [18] stellt den Nutzen von Statinen in der Hochrisikoprimärprävention (selbst bei Diabetikern) in Frage, weil darin kein Vorteil bezüglich Mortalität gefunden wurde. Nichtsdestotrotz empfehlen die US-Präventologen allen Männern ab 35 und Frauen ab 45 (also bis ins höchste Lebensalter), bei erhöhtem kardiovaskulärem Risiko die Lipidmessung und im Falle pathologischer Werte die Statintherapie [19].

Wenn Sie also infolge solcher Überlegungen vom Nutzen einer Medikation wie im angeführten Beispiel nicht überzeugt sind, nehmen Sie Abstand von Guidelineempfehlungen und setzen Sie das Medikament ab. Vielleicht nützen Sie dem Patienten damit sogar direkt, wie das folgende Beispiel illustriert.

2 Dieser Index wurde vor über 20 Jahren von Charlson et al. entwickelt, um die Einschätzung der Lebenserwartung nicht auf das chronologische Lebensalter, sondern auf die vorliegende Komorbidität des Probanden abzustützen. Ein multimorbider Patient hat, auch wenn er relativ jung ist, eine deutlich verkürzte Restlebenserwartung.

\section{Prävention und seine direkten Nebenwirkungen: Beispiel Antikoagulation}

Wie bei kurativen Therapien gibt es auch ganz konkrete unerwünschte Wirkungen präventiver Interventionen. Das Potential einer Schädigung kommt dabei manchmal demjenigen des Nutzens ungemütlich nahe, wie das folgende Beispiel illustriert: Die orale Antikoagulation wird verbreitet bei chronischem Vorhofflimmern oder rezidivierenden thromboembolischen Ereignissen (tiefe Venenthrombosen, Lungenembolien) eingesetzt, um cerebrovaskuläre Insulte respektive erneute Thrombosen/Lungenembolien zu vermeiden. Die in einer 2007 publizierten Metaanalyse [20] errechneten NNT für ein Jahr Behandlung lassen sich sehen: 40 in der Primärprävention, 14 in der Sekundärprävention und 32 für Patienten mit früherem CVI [21]. Leider sind aber auch die Blutungskomplikationen häufig: 2,3 von 100 Patienten erleiden pro Jahr eine Blutung, jede dritte gastrointestinal und jede siebte intrakraniell lokalisiert [22]. Bei älteren Patienten über 75 Jahren steigt diese Zahl auf 4,2 und klettert bei INR-Werten über 3,0 sogar bis auf 9 von 100 Behandelten pro Jahr, die eine Blutung erleiden [23]. Tabelle 5 zeigt die Bilanz:

Einer NNT von 14 bis 40 steht somit eine NNH von 11 bis 43 gegenüber. Wir sind also gut beraten, bei der präventiven Antikoagulation Höchstrisikopatienten oder solche mit früheren CVI zu identifizieren und generell den INR tief zu halten, ist doch die Schutzwirkung eines INR ab 1,8 derjenigen von höheren Werten ebenbürtig. Dieser Risikoselektion trägt die Entwicklung von Scores Rechnung, wie der CHADS-2-Score (Tab. 6) [24].

\section{Tabelle 6}

CHADS-2-Score [24]; Auswertung: ab Summe von zwei Punkten Antikoagulation empfohlen.

\begin{tabular}{llc}
\hline C & Congestive heart failure & 1 \\
\hline$H$ & Hypertension & 1 \\
\hline A & Age $>75$ & 1 \\
\hline$D$ & Diabetes & 1 \\
\hline S & Stroke or TIA & 2 \\
\hline
\end{tabular}

\section{Tabelle 7}

HAS-BLED-Score [25]; Auswertung: ab der Summe von drei Punkten engmaschige Überwachung und Überdenken der Indikation zur Antikoagulation empfohlen.

\begin{tabular}{lll}
\hline $\mathrm{H}$ & Hypertonie & 1 \\
\hline $\mathrm{A}$ & Abnorme Nieren- oder Leberfunktion & $1-2$ \\
\hline $\mathrm{S}$ & Schlaganfall & 1 \\
\hline $\mathrm{B}$ & Blutung & 1 \\
\hline $\mathrm{L}$ & Labiler INR-Wert & 1 \\
\hline $\mathrm{E}$ & Ältere Person über 65 & 1 \\
\hline $\mathrm{D}$ & Drogen oder Alkohol & $1-2$
\end{tabular}

Tabelle 5

Nutzen $[20,21]$ und Schaden $[22,23]$ von peroraler Antikoagulation, pro Person und Jahr Behandlung.

\begin{tabular}{|c|c|c|c|c|c|}
\hline Nutzen & Absolute Risikoreduktion & NNT (ein Jahr) & Schaden & Abs. Risiko & NNH (ein Jahr) \\
\hline Primärprävention & $2,5 \%$ & 40 & Blutungen alle & $2,3 \%$ & 43 \\
\hline Sekundärprävention & $7,0 \%$ & 14 & Alter $>75$ & $4,2 \%$ & 24 \\
\hline \multirow[t]{2}{*}{ St. n. CVI } & $3,1 \%$ & 32 & Alter $>75$, INR $<3$ & $2,7 \%$ & 37 \\
\hline & & & Alter $>75$, INR $>3$ & $9 \%$ & 11 \\
\hline
\end{tabular}


Der Benefit der Antikoagulation steigt mit jedem zusätzlichen Punkt des Scores [21]: So beträgt die absolute Risikoreduktion pro Jahr mit der Summe von einem Punkt 0,6\% (NNT = 167), mit 2 Punkten 1,3\% (NNT = 77), mit 3 Punkten 2,0\% (NNT = 50) und ab 4 Punkten $3,1 \%(N N T=32)$.

An ihrem letzten Jahreskongress präsentierten die Europäischen Kardiologen (ESC) eine Erweiterung des Scores um drei Risikofaktoren, die mit jeweils einem Scorepunkt bewertet werden: Vaskuläre Erkrankung, Altersdekade zwischen 65 und 74 und weibliches Geschlecht. Unter dem Strich qualifizieren sich (bei identischer «therapeutischer Schwelle» von zwei Punkten) also noch mehr Patienten für die Antikoagulation, benützte man diesen erweiterten Score. Die logische Folge, nämlich auch eine Risikoselektion nach Blutungsrisiken zu betreiben, führte zur Entwicklung des Blutungsrisiko-Scores HAS-BLED (Tab. 7) [25].

Die Hoffnung bleibt, dass mit Einführung der neuen Antikoagulantien (Faktor-II- und Faktor-X-Hemmer) bei erhaltenem Nutzen die Blutungsrisiken tiefer sein werden. Bisher liegen aber noch (zu) wenige Daten darüber vor, besonders bezogen auf die häufig betroffene ältere Population.

Das Ziel unserer Bemühungen bei der Nutzen-Risiko-Abwägung sollte also sein, den potentiellen Nutzen durch rigoroses Selektieren nach hohen Risiken zu steigern und gleichzeitig den potentiellen Schaden durch ebensolches Selektieren nach (und Vermeiden von) Behandlungsrisiken zu senken.

\section{Konklusion}

Eine mögliche Strategie, Leerläufe oder Schäden durch kardiovaskuläre Prävention im praktischen Alltag zu vermeiden, könnte also heissen:

- Selektion betreiben mittels Identifikation von Personen mit hohem kardiovaskulärem Risiko, zum Beispiel durch konsequente Risikoanamnese und den Gebrauch von Risiko-Scores (ESC-Score, AGLA-Score [26]). Damit sinkt auch die NNT.

- Eine «Gegenselektion» betreiben mittels Identifikation von Personen mit hohem Risiko, Nebenwirkungen gängiger Pharmaka zu erleiden: Wer ist anfällig für adverse Reaktionen? Zum Beispiel ist die Assoziation Alter - Niereninsuffizienz - Nebenwirkungen durch NSAR zu beachten. Damit sinkt die NNH.

- Die morbiditätsabhängige Lebenserwartung einschätzen (zum Beispiel anhand von morbiditätsabhängigen Indices wie dem Charlson-Index [16]) und im Zweifelsfall den Mut zum Verzicht aufbringen. Erlebt der Hochbetagte die Wirkung einer Intervention, eines Medikamentes mit grosser Wahrscheinlichkeit?

- Polypharmazie vermeiden: Bei allen Medikamentenlisten, die mehr als fünf Präparate enthalten, jedes einzelne Medikament der Frage nach der Indikation unterziehen: Ist es wirklich unverzichtbar? Überwiegt der Nutzen eines Medikamentes denjenigen der anderen bei weitem (siehe auch Beispiel im Kapitel «Grenznutzen und ist deshalb zu bevorzugen? Übersteigt sein erwarteter Nutzen das Risiko, dass es in Kombination mit den anderen Präparaten Schaden anrichtet?

- Fazit in vier Worten: Manchmal ist weniger mehr!

\section{Danksagung}

Ich danke Bruno Kissling und Peter Tschudi herzlich fürs kritische Gegenlesen.

\section{Literatur}

1 www.woncaeurope.org/definition.

2 www.sgam.ch/initiative.

3 Weingarten $M$, Matalon A. The ethics of basing community prevention in general practice. J Med Ethics. 2010;36:138-41.

$4 \mathrm{http}: / / \mathrm{ktclearinghouse.ca/cebm/glossary/nnt}$

5 Yarnall KS, Pollack KI, Ostbye T, Kause KM, Michener JL. Primary Care: Is there enough time for prevention? Am J Public Health. 2003;93:635-41.

6 Adams J, White M. When the population approach to prevention puts the health of individuals at risk. Int J Epidemiol. 2005;34:40-4.

7 Fugelli P. The zero-vision: Potential side effects of communicating health perfection and zero risk. Pat Educ and Counseling. 2006;60:267-71.

8 Neuner-Jehle S. Wieviel Risiko erträgt der Mensch? PrimaryCare. 2008; 13:253-6

9 Jorge Luis Borges (1899-1986); das Gedicht ist kurz vor seinem Tod entstanden.

10 Parkes G, Greenhalgh T, Griffin M, Dent R. Effect on smoking quit rate of telling patients their lung age: the Step2quit randomised controlled trial. BMJ. 2008;336:598-600.

11 Starfield B et al. The concept of prevention: a good idea gone astray? J Epidemiol Community Health. 2008;62:580-3.

12 Kühlein T, Sghedoni D, Visentin G, Gérvas J, Jamoulle M. Quaternäre Prävention, eine Aufgabe für Hausärzte. PrimaryCare. 2010;18:350-4

13 BMJ. 2000; 4(321):1107-11.

14 http://www.uni-protokolle.de/Lexikon/Gossensche_Gesetze.html.

15 Walter LC, Covinsky KE. Cancer screening in elderly patients: a framework for individualized decision making. JAMA. 2001;285(21):2750-6.

16 Charlson ME et al. J Chronic Dis. 1987;40:373-83.

17 Shepherd et al. Lancet. 2002;23;360(9346):1623-30.

18 Ray K et al. Statins an all-cause mortality in high-risk primary prevention. Arch Intern Med. 2010;170(12):1024-31.

19 U.S. Preventive Services Task Force. Guide to clinical preventive services. http://www.ahrq.gov/clinic/cps3dix.htm.

20 Hart RG et al. Ann Intern Med. 2007;146:857-67.

21 Singer $D$ et al. The net clinical benefit of warfarin anticoagulation in atrial fibrillation. Annals Intern Med. 2009;151:297-305.

22 Trullas-Villa JC. J Thromb Thrombolysis. 2009; doi 10.1007/s11239-009-0311-9.

23 Derry S, Loke YK. BMJ. 2000;321:1183-7.

24 American College of Cardiology; American Heart Association Task Force; European Society of Cardiology Committee for Practice Guidelines; European Heart Rhythm Association; Heart Rhythm Society et al: ACC/AHA/ESC 2006 guidelines for the management of patients with atrial fibrillation: 2006;8:651-745.

25 Guidelines for the managment of atrial fibrillation. Eur Heart J. 2010; doi:10.1093/eurheartj/ehq278.

26 http://www.agla.ch/content/rechner.php?l=1; http://www.heartscore.org/eu/low/Pages/Welcome.aspx.

\section{Korrespondenz:}

Dr. med. Stefan Neuner-Jehle, MPH

Facharzt Innere Medizin FMH

Institut für Hausarztmedizin und Versorgungsforschung

Pestalozzistrasse 24

8091 Zürich

sneuner@bluewin.ch 